\title{
Therapeutic Evaluation of Computed Tomography Findings for Efficacy of Prone Ventilation in Acute Respiratory Distress Syndrome Patients with Abdominal Surgery
}

\author{
Masayuki Akatsuka ${ }^{1,2^{*}}$, Hiroomi Tatsumi ${ }^{1}$, Naoya Yama ${ }^{3}$, Yoshiki Masuda ${ }^{1}$ \\ 1 Department of Intensive Care Medicine, Sapporo Medical University School of Medicine, Sapporo, Hokkaido, Japan \\ 2 Department of Anaesthesiology, Sapporo Medical University School of Medicine, Sapporo, Hokkaido, Japan \\ 3 Department of Diagnostic Radiology, Sapporo Medical University School of Medicine, Sapporo, Hokkaido, Japan
}

\begin{abstract}
Introduction: In Acute Respiratory Distress Syndrome (ARDS), the heterogeneity of lung lesions results in a mismatch between ventilation and perfusion, leading to the development of hypoxia. The study aimed to examine the association between computed tomographic (CT scan) lung findings in patients with ARDS after abdominal surgery and improved hypoxia and mortality after prone ventilation. Material and Methods: A single site, retrospective observational study was performed at the Sapporo Medical University School of Medicine, Sapporo, Hokkaido, Japan, between 1st January 2004 and 31st October 2018. Patients were allocated to one of two groups after CT scanning according to the presence of ground-glass opacity (GGO) or alveolar shadow with predominantly dorsal lung atelectasis (DLA) on lung CT scan images. Also, Patients were divided into a prone ventilation group and a supine ventilation group when the treatment for ARDS was started. Results: We analyzed data for fifty-one patients with ARDS following abdominal surgery. CT scans confirmed GGO in five patients in the Group A and in nine patients in the Group $B$, and DLA in 17 patients in the Group A and nine patients in the Group B. Both GGO and DLA were present in two patients in the Group A and nine patients in the Group B. Prone ventilation significantly improved patients' impaired ratio of arterial partial pressure of oxygen to fraction of inspired oxygen from $12 \mathrm{~h}$ after prone positioning compared with that in the supine position. Weaning from mechanical ventilation occurred significantly earlier in the Group $A$ with DLA vs the Group B with DLA $(P<0.001)$. Twenty-eight-day mortality was significantly lower for the Group A with DLA vs the Group $B$ with DLA $(P=0.035)$. Conclusions: These results suggest that prone ventilation could be effective for treating patients with ARDS as showing the DLA.
\end{abstract}

Keywords: prone position, computed tomography, acute respiratory distress syndrome

Received: 27 September 2019 / Accepted: 15 January 2020

\section{INTRODUCTION}

Acute respiratory failure after abdominal surgery for conditions such as pan-peritonitis frequently necessitates long-term mechanical ventilation, and treatment often ends in failure $[1,2]$. In particular, respiratory failure after abdominal surgery is known to cause respiratory damage both directly, when intra-abdominal infection or invasion affect scans the lungs via the diaphragm, and indirectly, mediated by the bloodstream [3]. Thoracic X-rays and the $\mathrm{PaO}_{2} / \mathrm{FiO}_{2}(\mathrm{P} / \mathrm{F})$ ratio are used in the diagnosis of acute respiratory distress syn- drome (ARDS), but lesions in the injured lungs may encompass a variety of different conditions. In ARDS, the heterogeneity of lung lesions results in a mismatch between ventilation and perfusion, leading to the development of significant hypoxia. Prone ventilation may potentially improve this ventilation-perfusion mismatch [4], but has not as yet been fully investigated. The present comparative investigation of the association between computed tomography (CT scan) findings from patients who had developed ARDS after abdominal surgery and improvements in hypoxia as a result of prone ventilation was therefore performed. 
The study aimed to examine the association between computed tomographic lung findings in patients with ARDS after abdominal surgery and improved hypoxia and mortality after prone ventilation.

\section{- MATERIALS AND METHODS}

This study was approved by the Institutional Review Board of Sapporo Medical University (Authorized number 302-156).

The single site retrospective study investigated patients admitted to the intensive care unit (ICU) in the hospital between 1st January 2004 and 31st October 2018.

\section{Subjects}

\section{Inclusion Criteria}

Participants were patients admitted to the intensive care unit (ICU) in the hospital between 1st January 2004 and 31st October 2018 who had developed ARDS following surgery for intra-abdominal infection and who had undergone lung CT scan on admission to the ICU. ARDS was developed and diagnosed following the criteria of the Berlin Definition [5] after being admitted to the ICU.

\section{Exclusion Criteria}

The patients who have less than 72 hours of mechanical ventilation and who is under 15 years old were excluded.

Subsequently, patients were divided into two groups: Group A that underwent prone ventilation within twenty four hours of the start of mechanical ventilation following ICU admission, and Group B, a Group B that did not.

\section{Classification of lung CT Scan findings}

Images from lung $\mathrm{CT}$ scan performed on admission to the ICU were evaluated by a single radiologist, and classified into two types according to the following procedure.

Six lung CT scan images were used for evaluation: $5 \mathrm{~cm}$ above the tracheal bifurcation, $5 \mathrm{~cm}$ below the tracheal bifurcation, and $5 \mathrm{~cm}$ above the diaphragm, covering each of the right and left lungs. Patients were categorised as showing either increasing ground glass opacity (GGO) or alveolar shadows with predominantly dorsal lung atelectasis (DLA) if these findings were evident in at least 3 of the 6 images

\section{Mechanical ventilation}

Respiration was managed so as to preserve spontaneous respiration. The ventilator mode used was pressure support ventilation. Blood gas analysis was performed every 4 hours, and support pressure was regulated to maintain $\mathrm{PaCO}_{2}$ at 35-50 mmHg. The fraction of inspired oxygen (FiO2) was regulated to maintain $\mathrm{PaO}_{2}$ $\geq 60 \mathrm{mmHg}$. The positive end-expiratory pressure (PEEP) value was set as recommended by the ARDS Network using allowable combinations of $\mathrm{FiO} 2$ and PEEP [6]

Sedation was carried out with fentanyl continuous infusion combined with continuous infusion of midazolam (0.03-0.06 mg/kg/h), propofol (0.5-3 mg/kg h), or dexmedetomidine $(0.2-0.7 \mu \mathrm{g} / \mathrm{kg} / \mathrm{h})$, and was regulated to maintain the patient at between -1 and -2 on the Richmond Agitation Sedation Scale. Prone ventilation did not involve any particular variation in sedation type or dosage.

\section{Prone method}

Prone ventilation was carried out using an air-cushioned bed. Patients were moved into the prone position under mechanical ventilation following the method previously reported [7]. An air-floating bed was used for changing patients to the prone position. At least five hospital staff members including medial doctors, intensive care nurses, and clinical engineers participated in each position change. Vital signs are monitored before and after the position change. Prone ventilation was continued for sixteen hours during which time blood gas analysis was performed every 4 hours.

The criteria for ending of prone ventilation were either that $\mathrm{PaO}_{2}$ was maintained at $\geq 80 \mathrm{mmHg}$ at $\mathrm{FiO}_{2}$ 0.5 for more than four hours after the patient had been returned to the supine position or there was no improvement in oxygenation compared with before the use of prone ventilation even after prone ventilation had been performed twice.

The criterion for ending prone ventilation was:

- $\mathrm{PaO}_{2}$ was maintained at $\geq 80 \mathrm{mmHg}$ at $\mathrm{FiO}_{2} 0.5$ for more than four hours after the patient had been returned to the supine position.

- There was no improvement in oxygenation after prone ventilation had been implemented twice in succession.

The criteria for prone ventilation were as follows:

- moderate ARDS according to the Berlin definition. 
- GGO, DLA and GGO+DLA in CT scan findings

- The discussion on treatment for the patients between the attending physician and the intensivists: whether prone ventilation could be effective considering vital signs and general conditions in patients.

The following data were obtained

- Age, sex,

- underlying diseases

- Acute physiology and chronic health evaluation (APACHE) II score,

- Sequential organ failure assessment (SOFA) scores on ICU admission

- Duration of stay in the ICU

- Duration of ventilation, outcome after 28 days and 90 days,

- PEEP value at the start of ventilation, and maximum PEEP value within 72 hours after the start of ventilation

- The number of ventilator-free days (VFDs) during which the patient was not attached to a ventilator

ICU-free days (IFDs) during which the patient was cared for in a ward other than the ICU.

To calculate the $\mathrm{P} / \mathrm{F}$ ratio in the Group $\mathrm{A}, \mathrm{PaO}_{2}$ and $\mathrm{FiO}_{2}$ were measured before patients were moved into the prone position and $12,24,48$, and 72 hours after the start of prone ventilation.

In the Group $\mathrm{B}, \mathrm{PaO}_{2}$ and $\mathrm{FiO}_{2}$ were measured 12 hours after the start of mechanical ventilation and at $12,24,48$, and 72 hours after the initial measurement.

To analyse the association between CT scan findings and the efficacy of prone ventilation, patients were divided into three groups based on lung CT scan images:

- those showing GGO (GGO group)

- those showing DLA (DLA group)

- those showing both (GGO + DLA group).

The $\mathrm{P} / \mathrm{F}$ ratio at the start of mechanical ventilation and 72 hours later were compared between the GGO and DLA groups in both Group A and Group B, and VFD, weaning rate from mechanical ventilation, and outcomes twenty days later, were also compared.

\section{Statistical analysis}

Changes over time in the $\mathrm{P} / \mathrm{F}$ ratio using repeatedmeasures analysis of variance were analysed.

The unpaired Student's t-test was used for comparisons between Group A and Group B and between the GGO and DLA groups.
Kaplan-Meier curves were produced for ventilation weaning rates over time.

Intergroup comparisons were made using the logrank test.

The level of significance was set at $\alpha=0.05$.

Values of $\mathrm{P}<0.05$ were regarded as significant.

\section{QRESULTS}

\section{Patient demographics and lung CT scan findings}

In total, fifty-one patients were admitted to the ICU during the study period with respiratory failure following abdominal surgery. Twenty-four underwent prone ventilation (Group A ) within 24 hours of being admitted to the ICU, and twenty-seven who underwent ventilation in the supine position (Group B). In Group A, mechanical ventilation was started within 24 hours of being admitted to the ICU followed by prone position. In Group B, mechanical ventilation was started within 24 hours of being admitted to the ICU.

Patient demographics are shown in Table 1.

Comparisons between Group A and Group B showed no significant differences in background CT scan characteristics were evident between the two groups for age or sex, APACHE II scores, SOFA scores on ICU admission or frequency of shock.

Upper gastrointestinal surgery was common in both the supine and Group A.

No significant difference in frequency of steroid administration was identified.

CT scan showed GGO in five patients in Group A and nine patients in Group B.

DLA occurred in 17 patients in Group A and nine patients in the Group B, and both (GGO + DLA) in two patient in the Group A and nine patients in Group B.

No significant differences in ventilation settings of PEEP level, peak pressure and respiratory rate were identified between the two group. The P/F ratio was $\leq$ 200 in both groups at the start of mechanical ventilation, meeting the diagnostic criteria for ARDS.

Comparison of changes in the $\mathrm{P} / \mathrm{F}$ ratio and weaning rate from mechanical ventilation

Table 1 shows changes in the $\mathrm{P} / \mathrm{F}$ ratio over time. No significant difference in the $\mathrm{P} / \mathrm{F}$ ratio at the start of the study was seen between both groups.

In the Group $\mathrm{A}$, the $\mathrm{P} / \mathrm{F}$ ratio rose significantly by 12 hours after the start of prone ventilation, and was sig- 
Available online at: www.jccm.ro

nificantly higher at 24, 48 and 72 hours compared with "prior to prone" ventilation, maintaining the improvement in oxygenation. Also, in Group B the P/F ratio was significantly elevated at 24,48 and 72 hours after the start of measurements compared with the value at the start of the study.

In Group $\mathrm{A}$, the $\mathrm{P} / \mathrm{F}$ ratio was significantly higher than the corresponding values for the Group B at each point.

\section{Duration of ventilation, duration of ICU stay, and outcome after 28 days and 90 days}

Group A had significantly more VFDs and IFDs (Table 1 ). The rate of weaning from mechanical ventilation at
The Journal of Critical Care Medicine 2020;6(1) • 35

twenty-eight days after the start of mechanical ventilation was also significantly higher in Group A than in Group B ( $\mathrm{P}=0.02)$ (Fig. 1). After 28 days, four patients in Group A had died (16.7\% mortality), and 10 of 27 patients in Group B had died (37.0\% mortality). This difference was not significant $(\mathrm{P}=0.127)$. However, the mortality rate after 90 days in Group A was significantly higher than that in Group B $(\mathrm{P}=0.048)$.

\section{Prone ventilation}

This technique was only applied once or twice (mean, $1.5 \pm 0.5$ times overall; $1.6 \pm 0.5$ times in the DLA group and $1.4 \pm 0.5$ times in the GGO group).

The mean time spent in the prone position was 16.1 \pm 0.8 hours.

\section{Table1. Patients' demographic data}

\begin{tabular}{|c|c|c|c|}
\hline & Group A & Group B & $\mathbf{P}$ \\
\hline Number & 24 & 27 & \\
\hline Age (year-old) & $71.0 \pm 11.2$ & $68.9 \pm 12.2$ & 0.508 \\
\hline Male / Female & $17 / 7$ & $18 / 9$ & 0.772 \\
\hline APACHE II & $21.0 \pm 5.8$ & $20.4 \pm 6.4$ & 0.734 \\
\hline SOFA & $7.3 \pm 3.1$ & $7.9 \pm 3.4$ & 0.469 \\
\hline Shock, n (\%) & $9(37.5)$ & $14(51.9)$ & 0.400 \\
\hline \multicolumn{4}{|l|}{ Surgical site } \\
\hline Upper tract & 15 & 12 & 0.162 \\
\hline Lower tract & 8 & 15 & \\
\hline Miscellaneous & 1 & 0 & \\
\hline \multicolumn{4}{|l|}{ Drug therapy } \\
\hline Steroid, n (\%) & $5(20.8)$ & $11(40.7)$ & 0.145 \\
\hline \multicolumn{4}{|l|}{ Ventilator settings at the start of the study } \\
\hline PEEP $(\mathrm{cmH} 2 \mathrm{O})$ & $10.3 \pm 2.3$ & $9.0 \pm 3.2$ & 0.116 \\
\hline Peak pressure $(\mathrm{cmH} 2 \mathrm{O})$ & $19.5 \pm 4.5$ & $18.3 \pm 5.7$ & 0.426 \\
\hline Respiratory rate & $21.5 \pm 6.8$ & $22.6 \pm 7.7$ & 0.584 \\
\hline \multicolumn{4}{|l|}{$\mathrm{PaO} 2 / \mathrm{FiO} 2$} \\
\hline At the start of mechanical ventilation & $118 \pm 41$ & $141 \pm 42$ & 0.030 \\
\hline \multicolumn{4}{|l|}{ At the time after the start of the study } \\
\hline $\mathrm{Oh}$ & $154 \pm 41$ & $156 \pm 30$ & 0.841 \\
\hline $12 \mathrm{~h}$ & $223 \pm 63$ & $166 \pm 46$ & $<0.001$ \\
\hline $24 \mathrm{~h}$ & $245 \pm 82$ & $182 \pm 49$ & 0.002 \\
\hline $48 \mathrm{~h}$ & $265 \pm 76$ & $202 \pm 46$ & $<0.001$ \\
\hline $72 \mathrm{~h}$ & $290 \pm 75$ & $226 \pm 65$ & 0.002 \\
\hline \multicolumn{4}{|l|}{ CT scan findings } \\
\hline $\mathrm{GGO}$ & 5 & 9 & 0.243 \\
\hline DLA & 17 & 9 & \\
\hline $\mathrm{GGO}+\mathrm{DLA}$ & 2 & 9 & \\
\hline Ventilator free days & $17.4 \pm 9.1$ & $11.5 \pm 9.8$ & 0.032 \\
\hline ICU free days & $14.4 \pm 9.1$ & $10.0 \pm 9.2$ & 0.095 \\
\hline 28-day mortality, n (\%) & $4(16.7)$ & $10(37.0)$ & 0.127 \\
\hline 90-day mortality, n (\%) & $5(20.8)$ & $13(48.1)$ & 0.048 \\
\hline
\end{tabular}


No serious complications such as accidental removal or kinking of the central venous catheter, tracheal tube or drains, or wound dehiscence occurred during prone ventilation. Mild reddening was identified around the cheekbones, iliac bones, and knees, but this resolved after patients were returned to the supine position.

Relationship between lung CT scan findings and efficacy of prone ventilation

Table 2 shows patients' data where DLA and GGO was shown on CT SCAN scans.

In total, 14 patients were classified as belonging to the GGO group on the basis of lung CT scan findings, of whom five underwent prone ventilation.

The DLA group contained 26 patients, 17 of whom underwent prone ventilation. The GGO and GLA groups showed no significant differences in age, sex, APACHE II score, SOFA score, surgical site, use of steroid, or ventilator settings at the start of this study.

In the GGO and DLA groups, no significant difference in the P/F ratio was apparent between Group A and Group B at the start of the study.

In patients with GGO, no significant difference in the $\mathrm{P} / \mathrm{F}$ ratio was seen in either Group A or Group B 72 hours after the start of this study.

In patients with DLA, the $\mathrm{P} / \mathrm{F}$ ratio was significantly higher in the Group A 72 hours after the start prone ventilation. There was no significant differences between Group A and Group B in the numbers of VFDs and IFDs for patients with GGO, but in patients with DLA, there was a significant differences between the two groups with regards to VFDs and IFDs (Table 3).

Weaning from mechanical ventilation in patients with DLA was also significantly earlier in Group A than in Group B $(\mathrm{P}<0.001)$, but no significant difference between Group A and Group B was identified for patients with GGO (P = 0.294) (Fig. 2).

The mortality rate after twenty eight days in patients in Group A with DLA was significantly lower than that in patients in Group B with DLA, but no significant difference between groups was seen for patients with GGO.

The outcome after twenty eight days in the DLA group was significantly higher in Group B than in Group A.

The mortality rate ninety days in Group A with DLA was also significantly lower than that in the Group B.
At 90 days, there was no significant difference in -mortality between the two groups with GGO (Table 3).

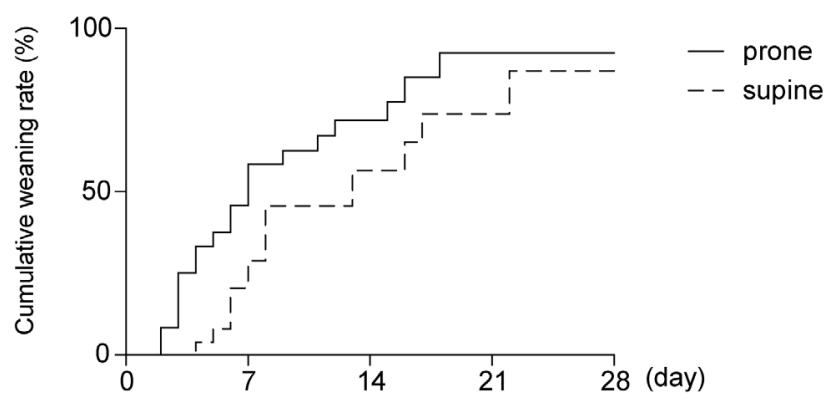

Fig. 1. Comparison of weaning rates from mechanical ventilation between the prone and supine ventilation groups in patients with intra-abdominal sepsis-induced ARDS. Cumulative weaning rate over 28 days was compared using the log-rank test. ARDS: acute respiratory distress syndrome

A

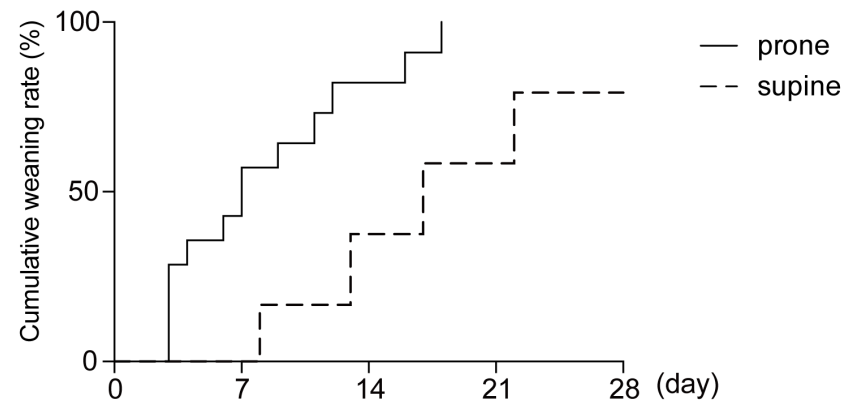

B

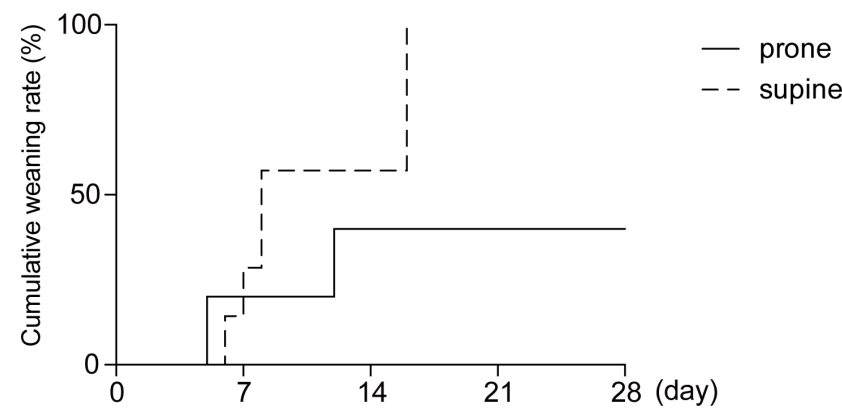

Fig. 2. Comparison of weaning rates from mechanical ventilation between the prone and supine ventilation groups in patients with DLA findings (A) and GGO findings (B) on CT scan. Cumulative weaning rate from mechanical ventilation in each group was compared using the logrank test. DLA: dorsal lung atelectasis; GGO: ground glass opacification; CT: computed tomography 
。์
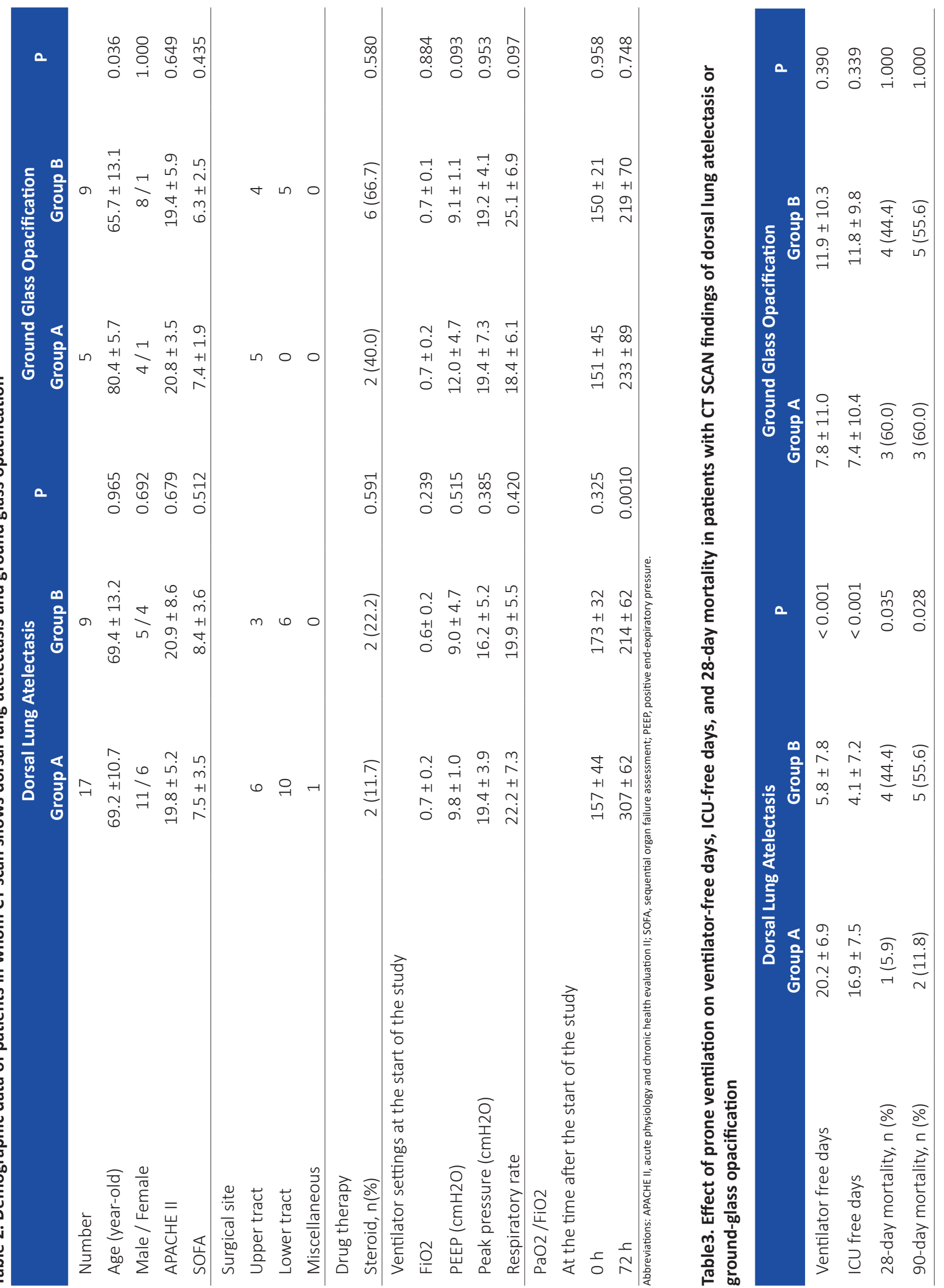


\section{DIsCUSSION}

Prone ventilation was performed for patients who developed acute respiratory failure after surgery for intraabdominal infection, and the association between the efficacy of prone ventilation and image findings from lung CT scan was examined. This technique was only applied once or twice because oxygenation could be improved. Of the 51 subjects in this study, twenty six (51.0\%) showed dorsal infiltration as the main finding on lung CT scan images, with only fourteen of the fifty one patients (27.5\%) showing mainly diffuse infiltration. Prone ventilation rapidly restored impaired oxygenation, and more than $50 \%$ of patients could be weaned off mechanical ventilation after $72 \mathrm{~h}$. A comparison of efficacy in terms of CT scan imaging findings showed that prone ventilation was clearly more effective in patients showing dorsal infiltration as the main finding compared with those with diffuse infiltration. The present results suggest that prone ventilation may be an effective method for treating patients with intra-abdominal infection who develop acute respiratory failure.

Acute respiratory failure associated with intra-abdominal infection is frequently extremely difficult to treat [1], and the mortality rate is reportedly high [2]. In an animal sepsis model of intra-abdominal infection, intra-abdominal fluid contained larger amounts of cytokines than seen in circulating blood [8]. These cytokines are continuously transferred into circulating blood, causing damage to the vascular endothelium of internal organs. In the lungs, this increases vascular permeability, increasing the volume of interstitial fluid and causing the appearance of diffuse infiltration on CT [9]. In particular, if vascular permeability increases in the dorsal region or on the surface of the diaphragm, where perfusion is greater because of gravity, intraabdominal fluid with a high concentration of inflammatory mediators may spread inflammation directly to the diaphragm concomitant with inflammation spreading from the diaphragm to the diaphragmatic surface of the lungs, causing the appearance of dorsal infiltration on CT. In addition, exposure of the diaphragm to inflammatory mediators may easily reduce its contractility [8]. The dorsal lungs are always vulnerable to deflation as a result of intra-abdominal pressure, but ventilation is normally maintained by appropriate contraction of the diaphragm during spontaneous respiration. Even during spontaneous respiration, however, atelectasis may easily occur if diaphragmatic function deteriorates [10]. The results of the present study suggest that, in acute respiratory failure associated with intra-abdominal infection, the spread of intra-abdominal inflammation via the surface of the diaphragm and inflammatory mediators in circulating blood may cause more dorsal infiltration in the lungs due to the action of gravity.

Prone ventilation has long been used to treat acute respiratory failure [11]. Randomised controlled trials carried out since 2000 have demonstrated its efficacy in restoring impaired oxygenation [12-14]. Although the mechanism whereby prone ventilation improves oxygenation is as yet unknown, the following hypotheses have been proposed: 1) improved diaphragm movement in the prone position [15];2) improved ventilation-perfusion mismatch [4]; 3) drainage of secretions that have collected in dorsal lung atelectasis [16]; 4) decrease in gravity-dependent increase of hydrostatic pressure [17]; and 5) improved trans-pulmonary pressure, which decreases due to abdominal pressure or increased lung mass [18]. On the basis of these mechanisms, it has been reported that prone positioning is more effective in patients with dorsal infiltration than in those with diffuse infiltration [19]. The greater improvement in hypoxia as a result of prone ventilation compared with the Group B may have been due to the fact that this study included more patients who showed dorsal atelectasis.

There is scope for debate concerning the improvement in outcomes as a result of prone ventilation. No such improvement in outcomes was evident in a randomised controlled trial carried out by Gattioni et al. (2001) [12], but in a subgroup analysis, severe cases with a $\mathrm{P} / \mathrm{F}$ ratio $\leq 150$ did show improved outcomes. A recent randomised controlled trial by Guerin et al.(2013) also found that prone ventilation improved outcomes for patients with ARDS and a P/F ratio $\leq 150$ [20]. A meta-analysis by Sud et al. (2010) likewise found that prone ventilation improved outcomes under conditions of a P/F ratio $\leq 140$ [21]. Such findings suggest that prone ventilation may have an important role to play as one method of treatment for severe respiratory failure with a $\mathrm{P} / \mathrm{F}$ ratio $\leq 150$.

In the present study, prone ventilation did not have any effect on improving outcomes. This may have been because the number of patients included in this study was too small to investigate outcomes, and only around half of the present patients (12 patients in each of Group A and Group B) had a P/F ratio $\leq 150$ at the 
time of inclusion, meaning that the study included few patients for whom prone ventilation could be expected to be effective.

Most previous studies of prone ventilation have used American-European Consensus Conference criteria to diagnosis of ARDS [22]. These diagnostic criteria stipulate the presence of bilateral infiltration on thoracic Xrays, but investigations of CT scan images have shown that a variety of conditions are included [23]. Few studies have addressed the association between the clinical efficacy of prone ventilation and findings on CT scan images in ARDS, which encompasses a heterogeneous range of conditions. In the present study, prone ventilation did not improve outcomes for patients with diffuse infiltration on CT scans, but did significantly improve outcomes in patients with dorsal infiltration. The great majority of patients with intra-abdominal infectionrelated ARDS also showed dorsal infiltration on CT scans. ARDS associated with intra-abdominal infection may thus be highly likely to progress to a condition in which prone ventilation may be effective, and prone ventilation may be a useful treatment option with mechanical ventilation.

In summary, most patients with ARDS associated with intra-abdominal infection showed dorsal atelectasis on CT scan, and prone ventilation enabled earlier weaning from mechanical ventilation. The present results indicate that prone ventilation may improve outcomes for patients with dorsal atelectasis on CT scan, and suggest prone ventilation as a useful treatment for patients with ARDS associated with intra-abdominal infection.

\section{DECLARATIONS OF INTEREST}

None to declare.

\section{DEFERENCES}

1. Wickel DJ, Cheadle WG, Mercer-Jones MA, et al. Poor outcome from peritonitis is caused by disease acuity and organ failure, not recurrent peritoneal infection. Ann Surg. 1997;225:74453.

2. Mustard RA, Bohnen JR, Rosati C, et al. Pneumonia complicating abdominal sepsis: an independent risk factor for mortality. Arch Surg. 1991;126:170-5.

3. Ware LB, Matthay MA. The Acute Respiratory Distress Syndrome. N Engl J Med. 2000;342:1334-49.

4. Pappert D, Rossaint R, Slama K, et al. Influence of positioning on ventilation-perfusion relationships in severe adult respiratory distress syndrome. Chest. 1994;106:1511-6.

5. Ranieri VM, Rubenfeld GD, Thompson BT, et al. Acute respiratory distress syndrome: Berlin Definition. JAMA. 2012;307:2526-33.

6. Acute Respiratory Distress Syndrome Network, Brower RG, Matthay MA, et al. Ventilation with lower tidal volumes as compared with traditional tidal volumes for acute lung injury and the acute respiratory distress syndrome. N Engl J Med. 2000;342:1301-8.

7. Masuda $Y$, Tatsumi $H$, Imaizumi $H$, et al. Effect of prone positioning on cannula function and impaired oxygenation during extracorporeal circulation. J Artif Organs. 2014;17:1069.

8. Fujimura N, Sumita S, Narimatsu E. Alteration in diaphragmatic contraCT scanility during septic peritonitis in rats: effect of polyethylene glycol-absorbed superoxide dismutase. Crit Care Med. 2000;28:2406-14.

9. Puybasset L, Cluzel P, Gusman P, et al. Regional distribution of gas and tissue in acute respiratory distress syndrome. I. Consequences for lung morphology. Intensive Care Med. 2000;26:857-69.

10. Froese $A B$, Bryan $A C$. Effect of anesthesia and paralysis on diaphragmatic mechanics in man. Anesthesiology. 1974;41:242-55.

11. Douglas WW, Rehder K, Beynen FM, et al. Improved oxygenation in patients with acute respiratory failure: the prone position. Am Rev Respir Dis. 1977;115:559-66.

12. Gattinoni L, Tognoni G, Pesenti A, et al. Effect of prone positioning on the survival of patients with acute respiratory failure. N Engl J Med. 2001;345:568-73.

13. Guerin C, Gaillard S, Lemasson S, et al. Effects of systematic prone positioning in hypoxemic acute respiratory failure a randomized controlled trial. JAMA. 2004;292:2379-87.

14. Alsaghir AH, Martin CM. Effect of prone positioning in patients with acute respiratory distress syndrome: a meta-analysis. Crit Care Med. 2008;36:603-9.

15. Krayer S, Rehder K, Vettermann J, et al. Position and motion of the human diaphragm during anesthesia-paralysis. Anesthesiology. 1989;70:891-8.

16. Piehl MA, Brown RS. Use of extreme position changes on acute respiratory failure. Crit Care Med. 1976;4:13-4.

17. Langer M, Mascheroni D, Marcolin R, et al. The prone position in ARDS patients. A clinical study. Chest. 1988;94:103-7.

18. Gattinoni L, Pelosi P, Vitale G, et al. Body position changes redistribute lung computed tomographic density in patients with acute respiratory failure. Anesthesiology. 1991;74:15-23.

19. Gainnier M, Michelet $P$, Thirion X, et al. Prone position and positive end-expiratory pressure in acute respiratory distress syndrome. Crit Care Med. 2003;31:2719-26.

20. Guérin C, Reignier J, Richard JC, et al. Prone positioning in 
40 - The Journal of Critical Care Medicine 2020;6(1)

severe acute respiratory distress syndrome. N Engl J Med. 2013;368:2159-68.

21. Sud S, Friedrich JO, Taccone P, et al. Prone ventilation reduces mortality in patients with acute respiratory failure and severe hypoxemia: systematic review and meta-analysis. Intensive Care Med. 2010;36:585-99.

22. Bernard GR, Atrigas A, Brigham KL, et al. The American-
Available online at: www.jccm.ro

European Consensus Conference on ARDS: Definitions, mechanisms, relevant outcomes, and clinical trial coordination. Am J Respir Critical Care Med. 1994;149:818-24.

23. Gattinoni L, Mascheroni D, Torensin A, et al. Morphological response to positive and expiratory pressure in acute respiratory failure. Computerized tomography study. Intensive Care Med. 1986;12:137-42. 